\title{
Método de Agrupamento Iterativo Baseado em Curvas Principais
}

\author{
Franciele A. Ferreira* André A. Braga ${ }^{* *}$ \\ Danton D. Ferreira ${ }^{* * *}$ Bruno H. G. Barbosa ${ }^{* * * *}$ \\ * Departamento de Automática, Universidade Federal de Lavras, MG, \\ (e-mail: franciele.ferreira1@posgrad.ufla.br). \\ ** Departamento de Automática, Universidade Federal de Lavras, MG, \\ (e-mail: aabraga@posgrad.ufla.br). \\ *** Departamento de Automática, Universidade Federal de Lavras, MG, \\ (e-mail:danton@ufla.br). \\ **** Departamento de Automática, Universidade Federal de Lavras, \\ $M G,(e-m a i l: b r u n o h @ u f l a . b r)$.
}

\begin{abstract}
The Principal Curves are one-dimensional and have high data representation capacity, which makes them a robust pattern recognition technique. This work presents an iterative clustering method using the Principal Curves technique. In the proposed method, initially and through the k-means algorithm, curves are allocated in each of the clusters found and, iteratively, the distance from each point to the curves is calculated, associating the point with the nearest curve. This process is performed iteratively until the convergence of the algorithm. The method was applied to synthetic databases and the results compared to those of the k-means algorithm. The proposed method obtained results better than or equal to that of k-means mainly in databases with curvilinear distribution. It was identified a certain sensitivity according to the initialization of the method showing the need to optimize its initial parameters.

Resumo: As Curvas Principais são unidimensionais e possuem alta capacidade de representação dos dados o que as torna uma técnica robusta de reconhecimento de padrões. Este trabalho apresenta um método de agrupamento de dados iterativo utilizando a técnica de Curvas Principais. No método proposto, inicialmente e por meio do algoritmo k-means, curvas são alocadas em cada um dos k agrupamentos encontrados e, de forma iterativa, a distância de cada ponto às curvas é calculada, associando o ponto à curva mais próxima. Este processo é realizado iterativamente até a convergência do algoritmo. O método foi aplicado a bases de dados sintéticas e os resultados comparados com os do algoritmo k-means. O método proposto obteve resultados melhores ou iguais ao do k-means principalmente em bases de dados com distribuição curvilínea. Identificou-se uma certa sensibilidade de acordo com a inicialização do método mostrando a necessidade de otimizar seus parâmetros iniciais.
\end{abstract}

Keywords: Principal Curves; Clustering; k-means; k-segments; Pattern Recognition.

Palavras-chaves: Curvas Principais; Agrupamento de Dados; k-means; k-segmentos; Reconhecimento de Padrões.

\section{INTRODUÇÃO}

Apresentado por Hastie and Stuetzle (1989) como uma generalização não linear da técnica de análise de componentes principais, as Curvas Principais (CP) foram definidas como curvas unidimensionais suaves que passam através de um conjunto multidimensional gerando uma representação unidimensional e não linear dos dados.

Hastie and Stuetzle (1989) ainda define formalmente curvas principais como aquelas curvas suaves que são autoconsistentes para uma distribuição ou conjunto de dados. A propriedade de autoconsistência significa que, para cada ponto selecionado na curva principal, a média da coleção de pontos de dados que projetam nesse ponto coincidirá com o ponto selecionado na curva.
Uma outra definição para curvas principais é apresentada por Kegl et al. (2000). Segundo os autores curvas principais são curvas contínuas de um determinado comprimento que minimizam a distância quadrada esperada entre a curva e os pontos do espaço escolhidos aleatoriamente de acordo com uma dada distribuição.

Tais características conferem a CP alta capacidade de representação de dados, o que faz com que ela seja uma técnica robusta de reconhecimento de padrões, para as quais podem ser encontradas na literatura diversas aplicações, como Ferreira et al. (2013) e Ferreira et al. (2014), que utilizam o algoritmo de curvas para detecção e classificação de distúrbios. Ferreira et al. (2015) propõem um índice de desvio de qualidade de energia com a utilização de CP. Chang and Ghosh (1998) utilizam técnicas baseadas em CP e em Self-organizing maps (SOM) para realizar 
classificação supervisionada de dados. Há ainda aplicações em sonares, para classificação de navios propostas por Fernandez (2005); Fernandes et al. (2015); Oliveira and Souza Filho (2017) e na identificação de blocos de gelo por imagem de satélites Banfield and Raftery (1992).

Devido à sua aplicabilidade, diferentes estratégias de implementação de $\mathrm{CP}$ podem ser encontradas. O primeiro algoritmo de obtenção de CP foi apresentado por Hastie and Stuetzle (1989) e sofreu diversas modificações com o passar dos anos. Najeh and Nabli (2013) comentam que os atuais algoritmos de curvas principais usam a primeira componente principal dos dados como uma estimativa inicial da curva principal. Se destacam ainda, o algoritmo de linhas poligonais (PLA), proposto por Kegl et al. (2000) e o algoritmo proposto por Wang et al. (2008) em que é realizdo o aprendizado de $\mathrm{CP}$ baseado em redes neurais artificiais, que transforma aprendizado não supervisionado em aprendizado supervisionado com a projeção do conjunto de dados em linhas poligonais, com a utilização de algoritmos de CP já existentes.

Além dos algoritmos já apresentados, um outro algoritmo bastante utilizado para construção de curvas principais é o algoritmo k-segmentos, proposto por Verbeek et al. (2002). O algorimto se destaca devido à sua robustez, baixa suscetibilidade a mínimos locais, convergência garantida e capacidade de lidar com dados que interceptam a si mesmo, quando comparado com outros algoritmos, tal como o PLA, proposto por Kegl et al. (2000).

A extração das CP a partir do algoritmo k-segmentos é feita de maneira incremental, a partir de um segmento, tendo o número de segmentos aumentado e a curva reconstruída a cada inserção de um novo segmento. Com a utilização deste algoritmo, Moraes and Ferreira (2016) propõem uma técnica de agrupamento de dados baseada em CP. Ferreira et al. (2013) investiga como a CP pode ser aplicada ao problema de monitoramento da qualidade de energia em termos de análise, detecção e classificação de eventos. Dal Cortivo and Marques (2014) propõem um classificador que substitui as distâncias médias das classes pelas distâncias aos segmentos das curvas principais geradas em cada classe pelo algoritmo k-segmentos.

Neste trabalho é proposto um método de agrupamento iterativo baseado em curvas principais. O método se assemelha ao comportamento do algoritmo k-means porém aloca curvas ao invés de centróides nos clusters gerados e substitui as distâncias dos eventos aos centróides pela distâncias às curvas como medida de classificação. O método foi aplicado à duas bases de dados sintéticas uma com distribuição dos dados de forma curvilínea e a outra com distribuição em círculos.

O restante do trabalho está organizado da seguinte maneira. A Seção 2 é divida em duas partes, onde na Subseção 2.1 é apresenta a fundamentação teórica do algoritmo ksegmentos e na Subseção 2.2 o método proposto é descrito. Já na Seção 3 os resultados e discussões são apresentados e por fim na Seção 4 a conclusão dessa etapa da pesquisa bem como os próximos passos a serem realizados são apresentados.

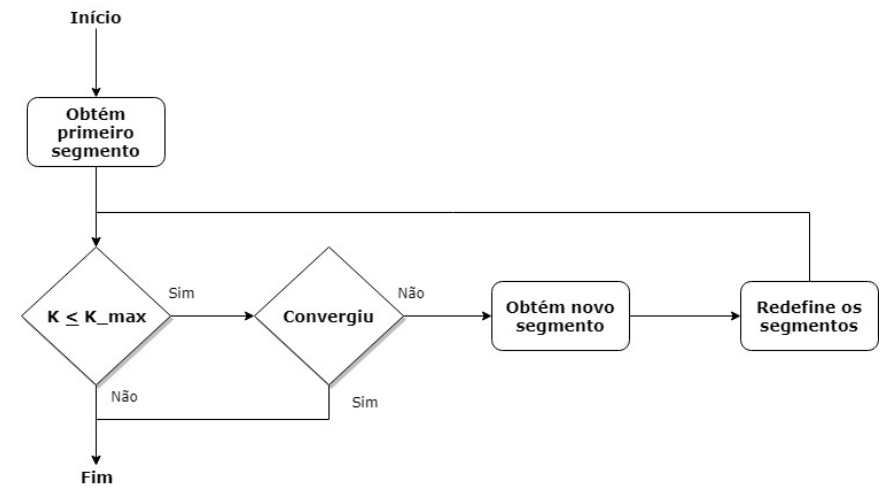

Figura 1. Fluxograma do algoritmo k-segmentos

\section{FUNDAMENTAÇÃO TEÓRICA}

\subsection{Algoritmo k-segmentos}

O algoritmo k-segmentos, foi proposto por Verbeek et al. (2002) para a extração de curvas principais. A robustez desse algoritmo e sua baixa susceptibilidade a mínimos locais faz com que ele se destaque em relação aos demais algoritmos de construção de curvas. A extração das CP a partir deste algoritmo é feita de maneira incremental, iniciando-se com apenas um segmento, sendo o número de segmentos aumentado progressivamente, com a otimização da curva a cada inserção, assim como mostrado no fluxograma da Figura 1.

A utilização desse algoritmo, porém, depende de certos parâmetros, tais como: a quantidade de segmentos que compõem a curva, $k$, e o coeficiente que determina como é feita a ligação entre diferentes segmentos, $\lambda$. No algoritmo original, o comprimento dos segmentos, por outro lado é fixo, com valor de $3 \sigma / 2$ sendo $\sigma^{2}$ a variância dos dados ao longo dos segmentos.

Braga et al. (2018) apresentaram um método de determinação automática da quantidade e comprimento de segmentos com a utilização da meta-heurística TeachingLearning Based Optimization, uma vez que foi observada a importância de se otimizar quantidade e comprimento de segmentos paralelamente, para a obtenção de CP com boa capacidade de representação de dados. Por outro lado, segundo Verbeek et al. (2001) os valores de $\lambda$ dentre $1 / 2$ e 2 apresentam bons resultados e a manutenção desse parâmetro inicial igual a 1 não traz prejuízos à curva gerada e sua capacidade de representação.

\subsection{Método proposto}

O método de agrupamento iterativo de dados proposto neste trabalho faz uso de CPs, extraídas pelo algoritmo $\mathrm{k}$-segmentos, para encontrar agrupamentos. O comportamento do método proposto se assemelha ao do algoritmo $k$-means no que diz respeito ao processo de agrupamento e convergência. Já em relação a inicialização, enquanto o algoritmo $k$-means inicializa centróides de forma aleatória o método proposto utiliza o próprio algoritmo $k$ means para definir inicialmente os agrupamentos e aloca curvas em cada um deles. Sendo assim, os eventos de cada agrupamento são associados à curva do agrupamento em questão. Após a inicialização, o método, de forma iterativa, 


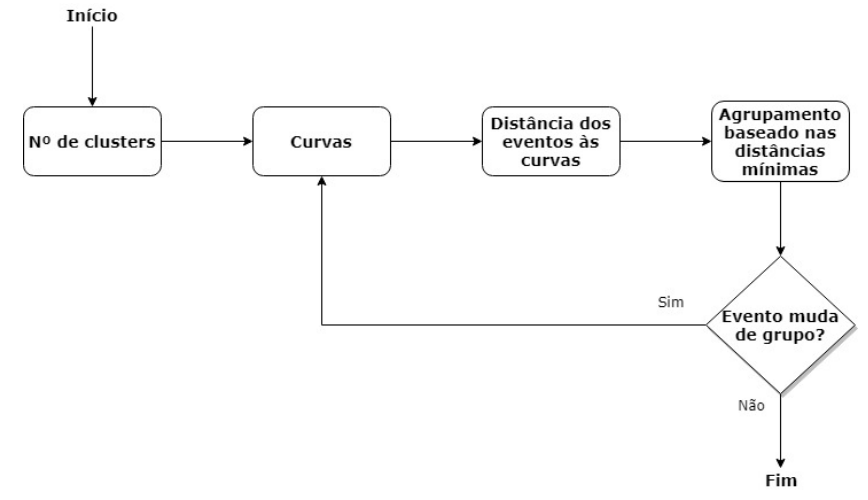

Figura 2. Fluxograma do método proposto

calcula a distância de cada evento às curvas alocadas e associa o evento à curva que ele está mais próximo. Caso identificado que um evento passou a ser associado a uma curva de um outro agrupamento, os eventos associados aos agrupamentos são atualizados e as curvas principais, por meio do algoritmo k-segmentos, são redefinidas. O método converge quando não há necessidade de associar o evento a uma curva diferente da que ele já se encontra associado.

As etapas do método proposto para encontrar os agrupamentos são descritas a seguir e a Figura 2 exibe o fluxograma do método:

(1) Define os clusters iniciais por meio do algoritmo kmeans.

(2) Aloca curvas através do algoritmo k-segmentos em cada um dos clusters gerados.

(3) Calcula a distância de cada evento às curvas dos clusters.

(4) Associa o evento ao cluster correspondente à curva que ele está mais próximo.

(5) Caso algum evento mude de cluster, calcula novas curvas para cada um dos clusters.

(6) Repete os passos de 2 à 5 até a convergência, ou seja, quando não há mais troca de agrupamento por evento.

\subsection{Comparação teórica entre os métodos}

O método proposto faz uso do algoritmo k-segmentos para a construção das curvas principais. De acordo com Verbeek et al. (2002), o algoritmo k-segmentos é uma extensão do algoritmo $k$-means que substitui os centróides por linhas e em seguida substitui as linhas por segmentos dela.

Sendo $S=\left\{s_{1}, s_{2}, \ldots, s_{k}\right\}$ o conjuto de linhas resultantes da primeira modificação do algoritmo, o objetivo segundo Verbeek et al. (2002), é encontrar k-linhas $s_{1}, \ldots, s_{k}$ que minimizam a distância total do quadrado de todos os pontos até a linha mais próxima deles sendo os pontos divididos em regiões de Voronoi $V_{i}$.

$$
\sum_{i=1}^{k} \sum_{x \in V_{i}} d\left(x, s_{i}\right)^{2}
$$

Segundo Gersho and Gray (1992), para um determinado conjunto finito de pontos $X \subset \Re^{d}$, o vetor médio $m$ desses pontos minimiza a função de distância ao quadrado:

$$
m=\arg \min _{\mu \in \Re^{d}} \sum_{x \in X}\|x-\mu\|^{2}
$$

Consequentemente:

$$
\sum_{x \in X} d(x, s)^{2} \leq \sum_{x \in X}\left\|x-x_{i}\right\|^{2}
$$

Sendo $x_{i}$ um ponto aleatório do conjunto $\mathrm{X}$, segundo Moraes et al. (2019), para o mesmo conjunto finito de pontos $X \subset \Re^{d}$, o segmento $s$ inclui $m$ e, portanto, a distância total do quadrado ao segmento é no máximo a distância total quadrada a $m$ :

$$
\sum_{x \in X}\|x-m\|^{2} \leq \sum_{x \in X}\|x-m\|^{2}
$$

Segundo Moraes et al. (2019), isso prova que usar um segmento para representar um conjunto de dados $X \subset \Re^{d}$ é melhor que um centróide (ou um neurônio). Uma vez que a curva principal é formada por k segmentos, usá-la é melhor que apenas um segmento.

\section{RESULTADOS E DISCUSSÃO}

De modo a verificar a capacidade de agrupamento de dados do método proposto, foram realizados testes em duas bases de dados sintéticas, cujos resultados foram comparados com os resultados obtidos pelo algoritmo de agrupamento $k$-means. Os testes foram divididos em duas etapas levando em consideração a otimização e a não otimização do comprimento e número de segmentos do algoritmo k-segmentos responsável por construir a curva principal.

Na primeira etapa, o número de segmentos foi definido experimentalmente por meio de vários testes com valores diferentes para este parâmetro cujo critério de seleção foi o valor que obteve melhor resultado. Já em relação ao comprimento do primeiro segmento utilizou-se o valor padrão do algoritmo original que corresponde a $3 \sigma / 2$ sendo $\sigma^{2}$ a variância dos dados ao longo dos segmentos.

Na segunda etapa foi utilizada a técnica de seleção automática do número e comprimento dos segmentos proposto por Braga et al. (2018). Essa técnica permitiu obter um valor mais adequado para o número e comprimento dos segmentos para a construção das curvas principais.

\subsection{Base de dados}

O método proposto foi aplicado à duas bases de dados sintéticas cada uma com duas classes. Uma das bases de dados possui os dados distribuídos em forma de duas parábolas com a concavidade para baixo e a outra base de dados possui os dados distribuídos em forma de dois círculos. Ambas bases de dados tiveram seus dados préprocessados onde foram eliminados dados repetidos e foi feita normalização na escala entre 0 e 1 . A quantidade de eventos por classe é apresentada na Tabela 1

\subsection{Parâmetros de entrada não otimizados}

A partir de vários testes com valores diferentes para o número de segmentos da curva principal foi possível 
Tabela 1. Número de eventos por classe

\begin{tabular}{cccc} 
Base de Dados & Classe A & Classe B & Total \\
\hline Parábolas & 59 & 59 & 118 \\
Círculos & 123 & 129 & 252 \\
\hline
\end{tabular}

determinar um valor que melhor representava a curva no meio dos conjuntos de dados.

Para ambas bases de dados considerou o número de segmentos $k=4$ e o comprimento do segmento $f=$ $3 \sigma / 2$. O resultado do agrupamento obtido tanto pelo método proposto quanto pelo algoritmo $k$-means, para a base de dados com representação de duas parábolas, são apresentados nas Figuras $3 \mathrm{a}$ e $3 \mathrm{~b}$ e as matrizes de confusão são apresentadas nas Tabelas $2 \mathrm{a}$ e $2 \mathrm{~b}$.

Já o resultado do agrupamento obtido para a base de dados com representação de dois círculos são apresentados nas Figuras $4 \mathrm{a}$ e $4 \mathrm{~b}$ e as matrizes de confusão apresentadas nas Tabelas $3 \mathrm{a}$ e $3 \mathrm{~b}$.

É possível observar que para a base de dados em formato de parábolas o método proposto com curvas principais conseguiu obter melhor agrupamento dos dados do que o algoritmo $k$-means já que a matriz de confusão do método proposto possui mais verdadeiros positivos e verdadeiros negativos do que a matriz de confusão do algoritmo $k$ means. Esse resultado era esperado visto que a técnica de CPs consegue modelar melhor os dados com distribuição curvilínea.

\begin{tabular}{ccc} 
& Classe A & Classe B \\
\hline Classe A & 31 & 28 \\
Classe B & 30 & 29 \\
\hline
\end{tabular}

(a) Algoritmo k-means Classe A Classe B

\begin{tabular}{lcc}
\hline Classe A & 47 & 12 \\
Classe B & 12 & 47 \\
\hline
\end{tabular}

(b) Método proposto

Tabela 2. Matriz de confusão base de dados de parábolas

Para a base de dados de círculos, de acordo com as matrizes de confusão representadas pelas Tabelas 3a e $3 \mathrm{~b}$ tanto o algoritmo $k$-means quanto o método proposto, conseguiram agrupar os dados com $100 \%$ de acerto.

Os resultados mostram que o algoritmo $k$-means teve resultados melhores com a base de dados de círculos uma vez que o algoritmo $k$-means consegue agrupar melhor os dados com distribuição circular.

\begin{tabular}{ccc} 
& Classe A & Classe B \\
\hline Classe A & 123 & 0 \\
Classe B & 0 & 129 \\
\hline
\end{tabular}

(a) Algoritmo k-means

\begin{tabular}{ccc} 
& Classe A & Classe B \\
\hline Classe A & 123 & 0 \\
Classe B & 0 & 129 \\
\hline
\end{tabular}

(b) Método proposto

Tabela 3. Matriz de confusão base de dados de círculos

\subsection{Parâmetros de entrada otimizados}

Com o objetivo de verificar se a otimização dos parâmetros de entrada do algoritmo k-segmentos influencia na eficácia

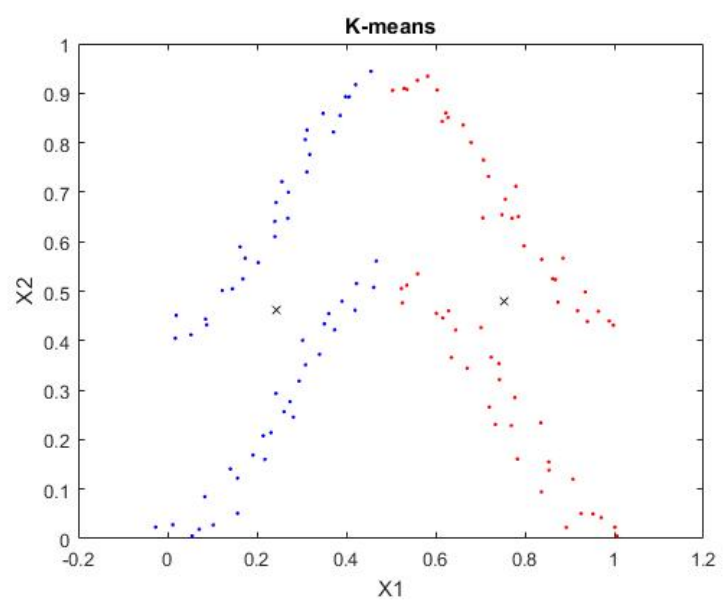

(a) K-means

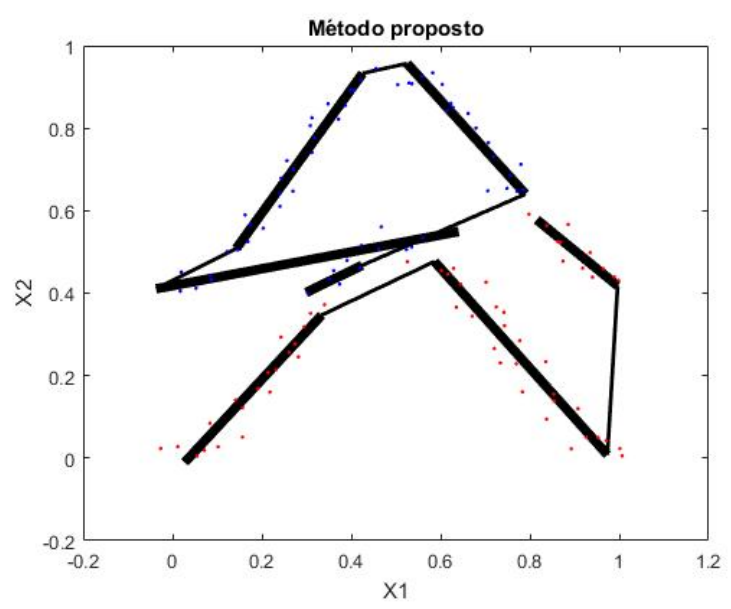

(b) Método proposto

Figura 3. Resultado do agrupamento sem otimização de parâmetros

do método proposto, utilizou-se a técnica de determinação automática da quantidade e comprimento de segmentos proposto por Braga et al. (2018) que utiliza a metaheurística Teaching-Learning Based Optimization.

O método proposto foi alterado incorporando a técnica de definição automática de parâmetros nas primeiras curvas geradas após a definição dos clusters iniciais pelo algoritmo $k$-means. As curvas geradas posteriormente até a convergência do método proposto utilizou os valores dos parâmetros já definidos pelas curvas inicias. Os valores dos parâmetros obtidos para as curvas de cada classe para as bases de dados de parábolas e de círculos são apresentados nas Tabela $4 \mathrm{a}$ e $4 \mathrm{~b}$

\begin{tabular}{ccc} 
& Classe A & Classe B \\
\hline$k$ & 6 & 5 \\
$f$ & 0.5689 & 0.6719 \\
\hline
\end{tabular}

(a) Base de dados de parábolas

\begin{tabular}{ccc} 
& Classe A & Classe B \\
\hline$k$ & 18 & 13 \\
$f$ & 0.5000 & 1.1208 \\
\hline
\end{tabular}

(b) Base de dados de círculos

Tabela 4. Valores dos parâmetros otimizados 


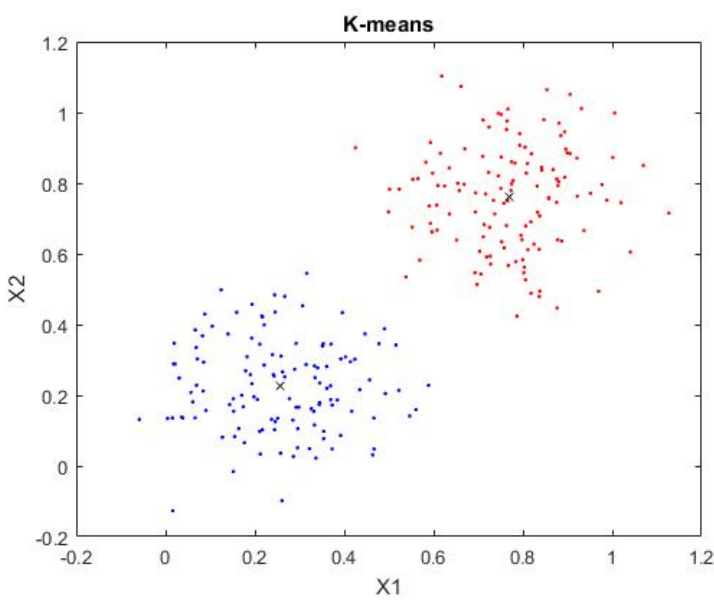

(a) K-means

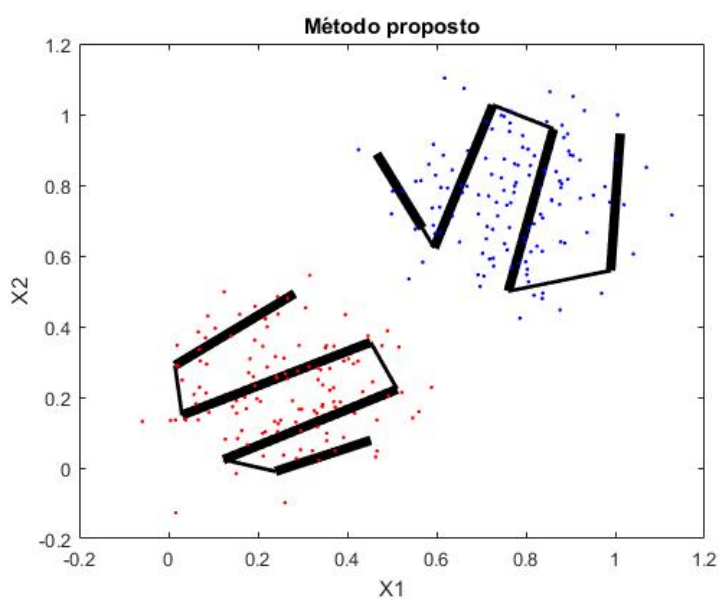

(b) Método proposto

Figura 4. Resultado do agrupamento sem otimização de parâmetros

O resultado do agrupamento obtido pelo método proposto com os parâmetros do algoritmo k-segmentos otimizados para as base de dados de parábolas e de círculos são apresentadas nas Figuras $5 \mathrm{a}$ e $5 \mathrm{~b}$ e as matrizes de confusão são apresentadas nas Tabelas 5a e 5b.

É possível verificar, comparando as matrizes de confusão representadas pelas Tabelas $2 \mathrm{~b}$ e $5 \mathrm{a}$, que houve uma melhora na acurácia do método proposto ao utilizar a técnica de otimização dos parâmetros do algoritmo k-segmentos, para a base de dados de parábolas. Comparando com a matriz de confusão do algoritmo $k$-means representada pela Tabela 2a, o método continuou obtendo melhores resultados para a base de dados de parábolas.

Já para a base de dados de círculos o método continuou agrupando os dados com $100 \%$ de acerto.

\section{CONCLUSÃO}

O método de agrupamento iterativo proposto nesse trabalho, conforme os resultados apresentados, conseguiu realizar o agrupamento dos dados com diferentes distribuições, curvilíneas e circulares.

É possível observar que para a base de dados de parábolas que possui a distribuição dos dados de forma curvilínea,

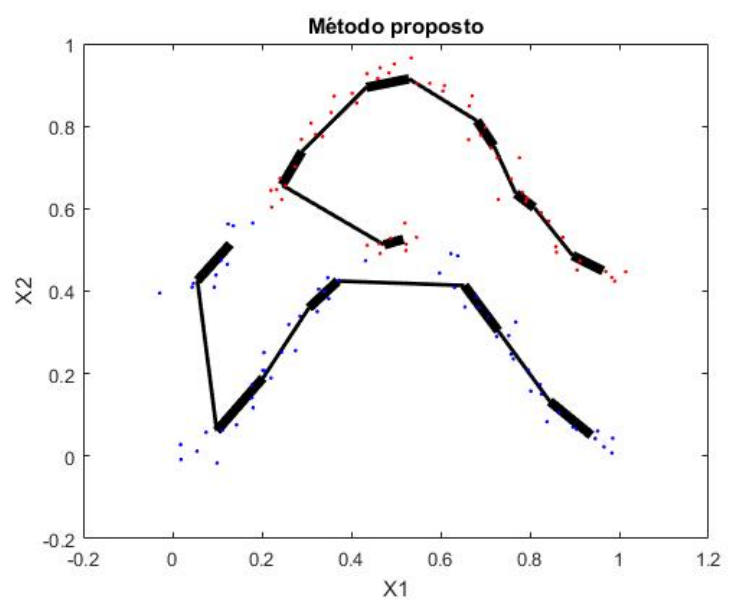

(a) Método proposto com base de dados de parábolas

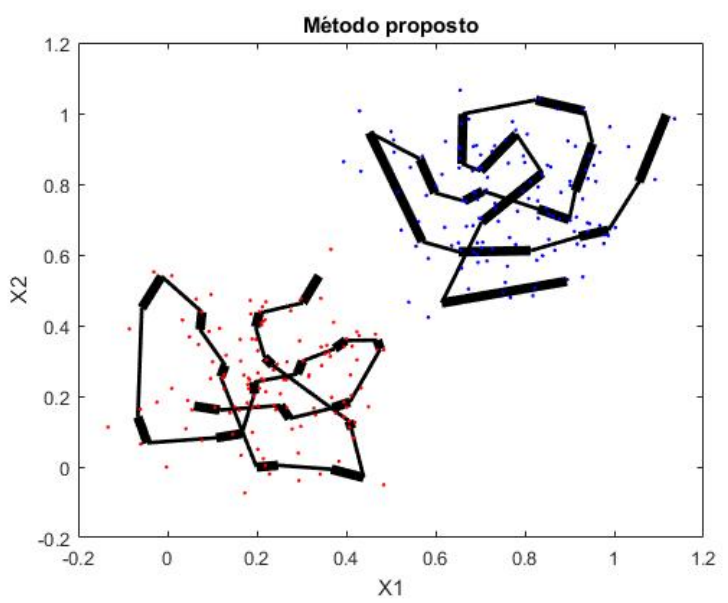

(b) Método proposto com base de dados de círculos

Figura 5. Resultado do agrupamento com otimização de parâmetros

\begin{tabular}{ccc} 
& Classe A & Classe B \\
\hline Classe A & 49 & 10 \\
Classe B & 8 & 51 \\
\hline
\end{tabular}

(a) Base de dados de parábolas

\begin{tabular}{ccc} 
& Classe A & Classe B \\
\hline Classe A & 123 & 0 \\
Classe B & 0 & 129 \\
\hline
\end{tabular}

(b) Base de dados de círculos

Tabela 5. Matriz de confusão do método proposto

o método proposto obteve melhores resultados do que o algoritmo $k$-means. Porém para a base de dados de círculos, tanto o método proposto quanto o algoritmo $k$ means conseguiram agrupar os dados com $100 \%$ de acerto.

Ao incorporar a otimização de parâmetros para o método proposto foi possível observar uma acurácia ainda maior do método proposto para a base de dados de parábolas que possui os dados com distribuição curvilínea.

Vale ressaltar que o método é dependente de uma boa inicialização pelo algoritmo $k$-means no momento da criação dos clusters iniciais. Foi observado ao longo dos testes que quando o algoritmo $k$-means agrupava os dados em clusters 
com acerto muito baixo, a acurácia do método proposto diminuía.

A implementação de formas para a redução da dependência da acurácia do método proposto quanto a sua inicialização serão os próximos passos a serem trabalhados nessa pesquisa juntamente com avaliação do método proposto utilizando base de dados reais.

\section{AGRADECIMENTOS}

Agradecemos a Fundação de Amparo à Pesquisa de Minas Gerais (FAPEMIG) e ao Consellho Nacional de Desenvolvimento Científico e Tecnológico ( $\mathrm{CNPq}$ ) pelo apoio financeiro com a pesquisa.

\section{REFERÊNCIAS}

Banfield, J.D. and Raftery, A.E. (1992). Ice floe identification in satellite images using mathematical morphology and clustering about principal curves. Journal of the American Statistical Association, 87(417), 7-16.

Braga, A.A., Ferreira, D.D., and Barbosa, B.H.G. (2018). Seleção automática de parâmetros iniciais do algoritmo k-segmentos com teaching-learning-based optimization. XXII Congresso Brasileiro de Automática, 1-7.

Chang, K. and Ghosh, J. (1998). Principal curve classifiera nonlinear approach to pattern classification. In 1998 IEEE International Joint Conference on Neural Networks Proceedings. IEEE World Congress on Computational Intelligence (Cat. No.98CH36227), volume 1, 695-700 vol.1.

Dal Cortivo, Z. and Marques, J. (2014). Classificação de dados amostrais baseado no algoritmo k-segmentos. In Proceeding Series of the Brazilian Society of Computational and Applied Mathematics, volume 02. doi: 10.5540/03.2014.002.01.0057.

Fernandes, T.S.P., de Oliveira e Souza Filho, J.B., and dos Santos Alves, E. (2015). Identificação de navios em sinais de sonar passivo por curvas principais. In C.J.A. Bastos Filho, A.R. Pozo, and H.S. Lopes (eds.), Anais do 12 Congresso Brasileiro de Inteligência Computacional, 1-6. ABRICOM, Curitiba, PR.

Fernandez, H.L. (2005). Classificação de Navios Baseada em Curvas Principais. Ph.D. thesis, Universidade Federal do Rio de Janeiro.

Ferreira, D.D., de Seixas, J.M., Duque, C.A., Cerqueira, A.S., and Ribeiro, P.F. (2014). A direct approach for disturbance detection based on principal curves. In 2014 16th International Conference on Harmonics and Quality of Power (ICHQP), 747-751.

Ferreira, D.D., de Seixas, J.M., Cerqueira, A.S., and Duque, C.A. (2013). Exploiting principal curves for power quality monitoring. Electric Power Systems Research, $100,1-6$.

Ferreira, D.D., de Seixas, J.M., Cerqueira, A.S., Duque, C.A., Bollen, M.H., and Ribeiro, P.F. (2015). A new power quality deviation index based on principal curves. Electric Power Systems Research, 125, 8 - 14.

Gersho, A. and Gray, R.M. (1992). Vector quantization and signal compression. Kluwer Academic Publishers.

Hastie, T. and Stuetzle, W. (1989). Principal curves. Journal of the American Statistical Association, 84(406), $502-516$.
Kegl, B., Krzyzak, A., Linder, T., and Zeger, K. (2000). Learning and design of principal curves. IEEE Transactions on Pattern Analysis and Machine Intelligence, $22(3), 281-297$.

Moraes, E.C.C. and Ferreira, D.D. (2016). A principal curve-based method for data clustering. In 2016 International Joint Conference on Neural Networks (IJCNN), 3966-3971.

Moraes, E.C.C., Ferreira, D.D., Vitor, G.B., and Barbosa, B.H.G. (2019). Data clustering based on principal curves. Advances in Data Analysis and Classification. doi:10.1007/s11634-019-00363-w. URL https://doi. org/10.1007/s11634-019-00363-w.

Najeh, T. and Nabli, L. (2013). Fault diagnosis using genetic algorithms and principal curves. International Journal of Research in Engineering and Technology, 02, 61-66.

Oliveira, T.C.C. and Souza Filho, J.B.d.O. (2017). Otimização de classificadores de sinais de sonar passivo baseado em curvas principais hierárquicas. In Anais do XIII Simpósio Brasileiro de Automação Inteligente, 569-574. Porto Alegre, RS.

Verbeek, J.J., Vlassis, N., and Kröse, B. (2001). A soft ksegments algorithm for principal curves. In Artificial Neural Networks - ICANN 2001, 450-456. Springer Berlin Heidelberg, Berlin, Heidelberg.

Verbeek, J., Vlassis, N., and Kröse, B. (2002). A ksegments algorithm for finding principal curves. Pattern Recognition Letters, 23(8), 1009 - 1017.

Wang, Y., Sun, Y., Liu, C., and Guo, Y. (2008). An algorithm for learning principal curves with explicit formulation based on neural network. In 2008 Congress on Image and Signal Processing, volume 1, 399-404. 